\title{
MAGNETIC BRAKING OF INTERSTELLAR CLOUDS
}

\author{
E.A. DORFI \\ Max-Planck-Institut für Astrophysik \\ Karl-Schwarzschild-Str. 1 \\ D-8046 Garching bei München, FRG
}

\begin{abstract}
We summarize the results of 3-D MHD calculations for a rotating and self-gravitating interstellar cloud. The angle between the axis of rotation and the magnetic field direction varies between $\pi / 2$ and 0 , the corresponding braking time-scales differ by roughly a factor of 3 .
\end{abstract}

\section{Introduction}

Observations of interstellar clouds clearly indicate that the formation, evolution and contraction of interstellar clouds is strongly influenced by the embedded magnetic fields. On theoretical grounds many properties of magnetic fields in the context of star formation are discussed in this issue and we concentrate on the problem of magnetic braking during the gravitational collapse of an interstellar cloud of $M_{\mathrm{cl}}=10^{4} M_{\odot}$. The radius is given by $R_{\mathrm{cl}}=6.510^{19} \mathrm{~cm}$ and hence, the density corresponds to $\rho_{\mathrm{cl}}=1.7310^{-23} \mathrm{~g} \mathrm{~cm}^{-3}$. The rotational velocity is set to the galactic angular velocity of $\Omega=10^{-15} \mathrm{sec}^{-1}$. The temperature of $T=100 \mathrm{~K}$ is kept fixed throughout the whole calculation. The cloud is embedded in a tenuous medium of $\rho_{\text {ext }}=0.1 \rho_{\mathrm{cl}}$ in pressure equilibrium. A homogeneous magnetic field of $3 \mu \mathrm{G}$ permeates the cloud and the interstellar medium and is inclined to the axis of rotation. These initial conditions yield a free fall time of $t_{f f}=1.6 \cdot 10^{7} \mathrm{yr}$ and an Alfvén crossing-time in the external medium of $R_{\mathrm{cl}} / v_{\mathrm{A} \text {,ext }}=3.2 \cdot 10^{6} \mathrm{yr}$.

\section{Results}

The development of bar-like or pancake-like, non-homologous structures is very common in these 3-dimensional calculations. Although the detailed evolution depends strongly on the orientation of the magnetic field the braking time differs by roughly a factor of 3 for different angles (cf. Fig. 1). In the case of an inclined or perpendicular magnetic field oscillatory motions occur on various scales and retrograde rotation is seen several times during the calculated temporal evolution which leads to an overall slower decrease of the total angular momentum contained in a sphere of radius $R_{\mathrm{cl}}$. The exerted magnetic torques rotate the total angular momentum vector towards the field direction. Therefore the final orientation of 
Total angular momentum

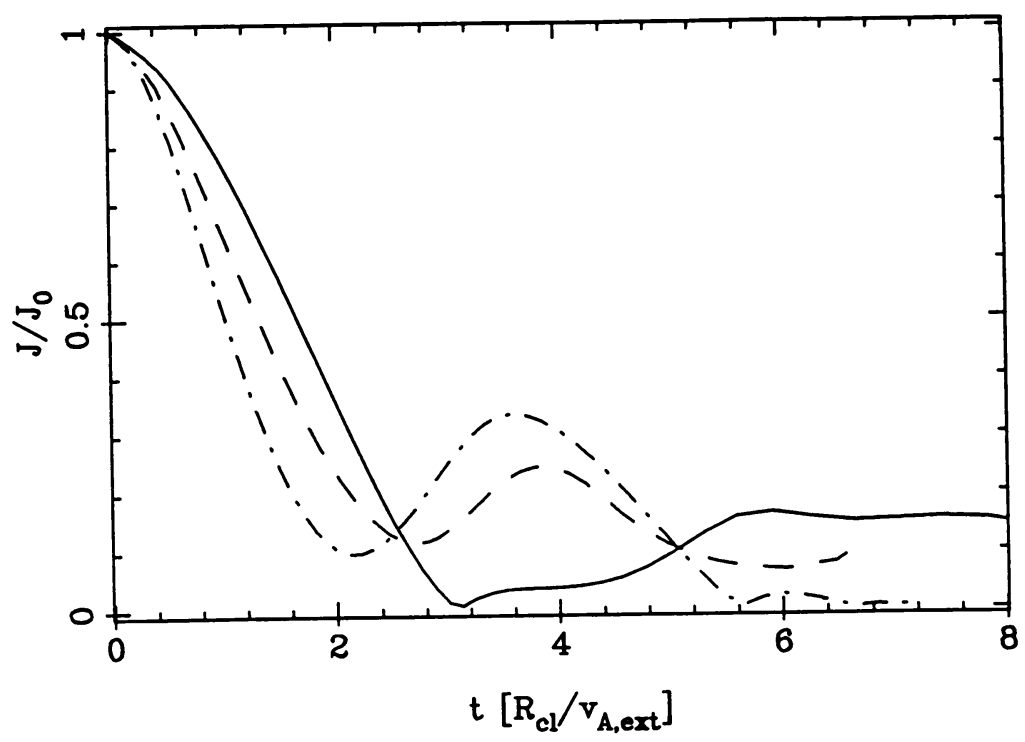

Figure 1: The temporal evolution of the total angular momentum for three different angles between the axis of rotation and the magnetic field The angular momentum is normalized to the initial value of $J_{0} / M_{\mathrm{cl}}=6.410^{24} \mathrm{~cm} \mathrm{sec}^{-1}$. The time is given in the dimensionless Alfvén crossing-time in the external medium of $R_{\mathrm{cl}} / v_{\mathrm{A}, \text { ext }}=$ $3.2 \cdot 10^{6} \mathrm{yr}$. The solid line corresponds to the aligned magnetic field i.e. $\theta=0$, the dashed line denotes an inclined magnetic field of $\theta=\pi / 4$. and the dashed-dotted line shows the perpendicular case of $\theta=\pi / 2$. The corresponding times of the first minima of these three curves are at $t_{\min }=2.10,2.75$ and 3.15 for the magnetic field angles of $\theta=\pi / 2, \pi / 4$ and 0 , respectively.

the rotation axis and the remaining angular momentum of the contracting cloud depend on the time when ambipolar diffusion decouples the magnetic field from the gas. After about $1.5 t_{f f}$ central condensations of $\rho \approx 100-1000 \rho_{\mathrm{cl}}$ are formed and we get a core-envelope structure. These cores containing a few $100 M_{\mathrm{cl}}$ have no significant velocities relative to the sound speed. The tenuous envelope contains most of the initial mass but its further contraction is inhibited by the magnetic forces. For a more detailled discussion we refer to Dorfi (1989).

\section{References}

Dorfi, E.A.: 1989, Astron. Astrophys., in press 